\title{
FCER2 Positive
}

National Cancer Institute

\section{Source}

National Cancer Institute. FCER2 Positive. NCI Thesaurus. Code C132231.

Indicates that FCER2 expression has been detected in a sample. 